\title{
Optical Energy Losses in Organic-Inorganic Hybrid Perovskite Light-Emitting Diodes
}

Xiaobo Shi, Yuan Liu, Zhongcheng Yuan, Xiaoke Liu, Yanfeng Miao, Jianpu Wang, Simone Lenk, Sebastian Reineke and Feng Gao

The self-archived postprint version of this journal article is available at Linköping University Institutional Repository (DiVA):

http:// urn.kb.se/ resolve?urn=urn:nbn:se:liu:diva- 151477

N.B.: When citing this work, cite the original publication.

Shi, X., Liu, Y., Yuan, Z., Liu, X., Miao, Y., Wang, J., Lenk, S., Reineke, S., Gao, F., (2018), Optical Energy Losses in Organic-Inorganic Hybrid Perovskite Light-Emitting Diodes, Advanced Optical Materials, 6(17), 1800667. https:/ / doi.org/ 10.1002/ adom.201800667

Original publication available at:

https:// doi.org/ 10.1002/adom.201800667

Copyright: Full Open Access

Publisher: John Wiley \& Sons 


\title{
Optical Energy Losses in Organic-Inorganic Hybrid Perovskite Light-Emitting Diodes
}

\author{
Xiao-Bo Shi, Yuan Liu, Zhongcheng Yuan, Xiao-Ke Liu, * Yanfeng Miao, Jianpu Wang, \\ Simone Lenk, Sebastian Reineke, and Feng Gao*
}

Light-emitting diodes (LEDs) based on organic-inorganic hybrid perovskites, in particular, 3D and quasi-2D ones, are in the fast development and their external quantum efficiencies (EQEs) have exceeded $10 \%$, making them competitive candidates toward large-area and low-cost light-emitting applications allowing printing techniques. Similar to other LED categories, light out-coupling efficiency is an important parameter determining the EQE of perovskite LEDs (PeLEDs), which, however, is scarcely studied, limiting further efficiency improvement and understanding of PeLEDs. In this work, for the first time, optical energy losses in PeLEDs are investigated through systematic optical simulations, which reveal that the 3D and quasi-2D PeLEDs can achieve theoretically maximum EQEs of $\approx \mathbf{2 5 \%}$ and $\approx \mathbf{2 0 \%}$, respectively, in spite of their high refractive indices. These results are consistent with the reported experimental data. This work presents primary understanding of the optical energy losses in PeLEDs and will spur new developments in the aspects of device engineering and light extraction techniques to boost the EQEs of PeLEDs.

easily tunable band gaps. ${ }^{[4-7]}$ LEDs based on such materials have been attracting increasing attention since the first roomtemperature perovskite LEDs (PeLED) developed in 2014. ${ }^{[13]}$ The development of PeLEDs has progressed very rapidly, realizing a record-high external quantum efficiency (EQE) of $11.7 \%$ in 2016. ${ }^{[14]}$

Similar to other LED categories, e.g., organic LEDs (OLEDs) ${ }^{[15]}$ and quantum$\operatorname{dot}^{[16,17]}$ LEDs, the EQE of PeLEDs is determined by the internal quantum efficiency (IQE) and light out-coupling efficiency $(\eta)$, which is expressed as

$\mathrm{EQE}=\eta \cdot \mathrm{IQE}=\eta \cdot \gamma \cdot \eta_{\mathrm{S} / \mathrm{T}} \cdot q_{\mathrm{eff}}$

The IQE is the product of the charge carrier balance $(\boldsymbol{\gamma})$, the fraction of excitons capable of radiative decay at room temperature $\left(\eta_{\mathrm{S} / \mathrm{T}}\right)$, and the effective radiative

Organic-inorganic perovskite materials have attracted extensive interest due to their fascinating semiconducting properties, such as high absorption coefficient, long carrier diffusion length, and small exciton binding energy. ${ }^{[1-3]}$ Moreover, these materials are easily processed via solution-based techniques, showing great potential applications in low-cost optoelectronic devices such as solar cells, ${ }^{[1-3]}$ light-emitting diodes (LEDs), ${ }^{[4-7]}$ lasers, ${ }^{[6-8]}$ and transistors. ${ }^{[9-11]}$ In the past few years, we have witnessed the rapid improvement of power conversion efficiency of perovskite solar cells, which reaches over $22 \%$ in 2016. ${ }^{[12]}$

In addition to their excellent properties for light absorbers, perovskite materials are also outstanding emitters with high color purity, high photoluminescent quantum yield (PLQY), and quantum yield $\left(q_{\text {eff }}\right)$. For $2 \mathrm{D}$ perovskite materials with relatively large binding energy, the $\eta_{\mathrm{S} / \mathrm{T}}$ could be unity when heavy atoms are involved in the organometallic complex (similar to that in phosphorescent materials ${ }^{[18]}$ ). For 3D perovskite materials with relatively small binding energy, the emission should be bandto-band transitions of free carriers in direct gap semiconductors, and the $\eta_{\mathrm{S} / \mathrm{T}}$ term should not be involved in the equation in this case. All in all, this equation shows that the EQE is largely influenced by the $\eta$ besides the IQE. Thus, studying the limits of $\eta$ is of great importance for achieving high-EQE PeLEDs.

As the fast development of PeLEDs, a conflict appears between the experimental and theoretical results. In general, the $\eta$ can be estimated according to the ray-optics theory, ${ }^{[19]}$
Dr. X.-B. Shi, Z. C. Yuan, Dr. X.-K. Liu, Dr. F. Gao

Department of Physics, Chemistry and Biology (IFM)

Linköping University

Linköping 58183, Sweden

E-mail: xiaoke.liu@liu.se; feng.gao@liu.se

Y. Liu, Dr. S. Lenk, Prof. S. Reineke

Dresden Integrated Center for Applied Physics and

Photonic Materials (IAPP) and Institute for Applied Physics

Technische Universität Dresden

Dresden 01187, Germany

The ORCID identification number(s) for the author(s) of this article can be found under https://doi.org/10.1002/adom.201800667.

DOI: 10.1002/adom.201800667

\section{Dr. X.-K. Liu}

State Key Laboratory of Luminescent Materials and Devices

South China University of Technology

Guangzhou 510640, P. R. China

Dr. X.-K. Liu

State Key Lab of Silicon Materials

Zhejiang University

Hangzhou 310027, P. R. China

Y. F. Miao, Prof. J. P. Wang

Key Laboratory of Flexible Electronics (KLOFE) \& Institute of Advanced

Materials (IAM) \& Jiangsu National Synergistic Innovation Center for

Advanced Materials (SICAM)

Nanjing Tech University (NanjingTech)

30 South Puzhu Road, Nanjing 211816, P. R. China 
which has been well acknowledged in OLEDs as a first approximation, that is

$\eta=\frac{1}{2 n^{2}}$

where $n$ is the refractive index of the emitting material. As for PeLEDs based on methylammonium lead iodide $\left(\mathrm{MAPbI}_{3}\right)$ and $\mathrm{NFPI}_{7}$ (the mixture of 1-naphthylmethylamine iodide, formamidinium iodide, and $\mathrm{PbI}_{2}$ with the molar ratio of 2:1:2), it would be $7.4 \%$ and $12.5 \%$ respectively because of their large refractive index of $\approx 2.6$ and $\approx 2.0$, respectively, at the emissive wavelength. The $\eta$ can be estimated using Equation (1) from experimental data based on the EQE and the PLQY with the assumption of good charge carrier balance $(\gamma=1)$. By using this method, a $\eta$ of $\approx 19.5 \%$ can be obtained for the NFPI ${ }_{7}$-based PeLEDs. ${ }^{[14]}$ Such a conflict implies that the optical processes in PeLEDs are quite different from those in the OLEDs, and more complicated than what the ray-optics theory can predict. Therefore, more precise optical simulations are highly desirable to understand the optical energy losses in PeLEDs and predict their theoretical EQE limits.

Herein, we systematically study the optical energy losses in PeLEDs through advanced optical simulations. Our results show that the maximum $\eta\left(\eta_{\max }\right)$ of $\mathrm{MAPbI}_{3}$-based 3D and $\mathrm{NFPI}_{7}$-based quasi-2D PeLEDs are $\approx 25 \%$ and $\approx 20 \%$, respectively. However, the 3D PeLEDs based on $\mathrm{MAPbI}_{3}$ with relatively larger refractive index show $\eta$ values that are more sensitive to the thickness of the emitting layer (EML), whereas the quasi-2D PeLEDs based on $\mathrm{NFPI}_{7}$ with relatively smaller refractive index show less thickness dependence. In addition, there is strong coupling between the waveguide mode and evanescent/ surface plasmon polariton (SPP) mode in PeLEDs with large refractive indices, resulting in new hybrid optical modes and different spatial power distribution inside the PeLEDs. These results suggest that further development of high-efficiency PeLEDs can be realized through device engineering or reducing the substrate and the hybrid optical modes using various light extraction technologies, ${ }^{[20,21]}$ e.g., attaching an index-matched hemisphere at the air/glass interface and forming microstructures at the $\mathrm{ZnO} / \mathrm{EML}$ interface.

We adopt the classical electromagnetic model to describe the electroluminescence in PeLEDs (details are described in the Experimental Section). In the classical electromagnetic model, the radiative decay of excitonic species are treated as an ensemble of classical electrical dipole antennas radiating electromagnetic power. ${ }^{[22-28]}$ The device structure used for simulations is shown in Figure 1a, consisting of indium tin oxide (ITO), zinc oxide ( $\mathrm{ZnO})$, perovskite EML, poly[(9,9dioctylfluorenyl-2,7-diyl)-co-(4,4-(N-(4-sec-butylphenyl)) diphenylamine)] (TFB), molybdenum oxide $\left(\mathrm{MoO}_{3}\right)$, and gold $(\mathrm{Au})$. Two widely reported green and near-infrared 3D perovskite materials, ${ }^{[13,29-31]}$ methylammonium lead bromide $\left(\mathrm{MAPbBr}_{3}\right)$ and $\mathrm{MAPbI}_{3}$, and the highest-efficiency quasi-2D perovskite $\mathrm{NFPI}_{7}{ }^{[14]}$ are chosen as the EMLs in this study. The refractive indices of $\mathrm{MAPbI}_{3}$ and $\mathrm{NFPI}_{7}$ are measured to be $\approx 2.6$ and $\approx 2.0$ at their emissive wavelengths $(700-850 \mathrm{~nm})$, respectively, and that is $\approx 2.2$ for $\mathrm{MAPbBr}_{3}$ at $450-550 \mathrm{~nm}$ (Figure $1 \mathrm{~b}$ ). The refractive indices of other materials used in this study are shown in Figure S1 in the Supporting Information. In consideration of the wide distribution of refractive indices of perovskite materials, various refractive index values (1.75-3.00) are studied.

The simulated dispersion diagrams of transverse magnetic (TM) polarized light are shown in Figure 2, which indicate how the radiated power changes with the energy and the in-plane wavevector. Each graph in Figure 2 is divided into three regions corresponding to the direct emission mode (Region 1, light into the air from the forward hemisphere direction), the substrate mode (Region 2, light trapped inside the glass substrate), and the waveguide/SPP mode (Region 3, light trapped inside the functional layers), respectively. The details of how to define the three regions are presented in the Experimental Section. As for the direct emission mode (Region 1) and the substrate mode (Region 2), their dispersion relation rarely changes with the refractive index of the EML. However, in Region 3, three continuous bright curves (namely $\mathrm{TM}_{0}, \mathrm{TM}_{1}$, and $\mathrm{TM}_{2}$ in the order from the right-bottom corner to the left-top corner) appear in Figure 2a, indicating different TM modes. As the refractive
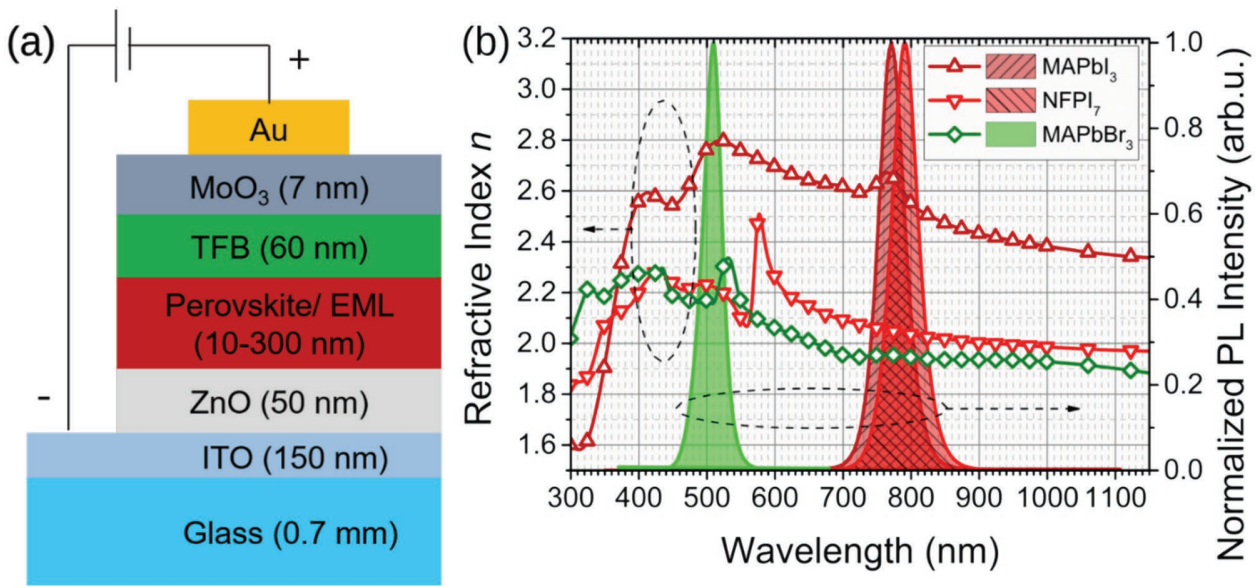

Figure 1. a) Schematic illustration of the device structure of the PeLEDs used for optical simulations. b) The refractive indices and photoluminescence spectra of the selected perovskite materials. 

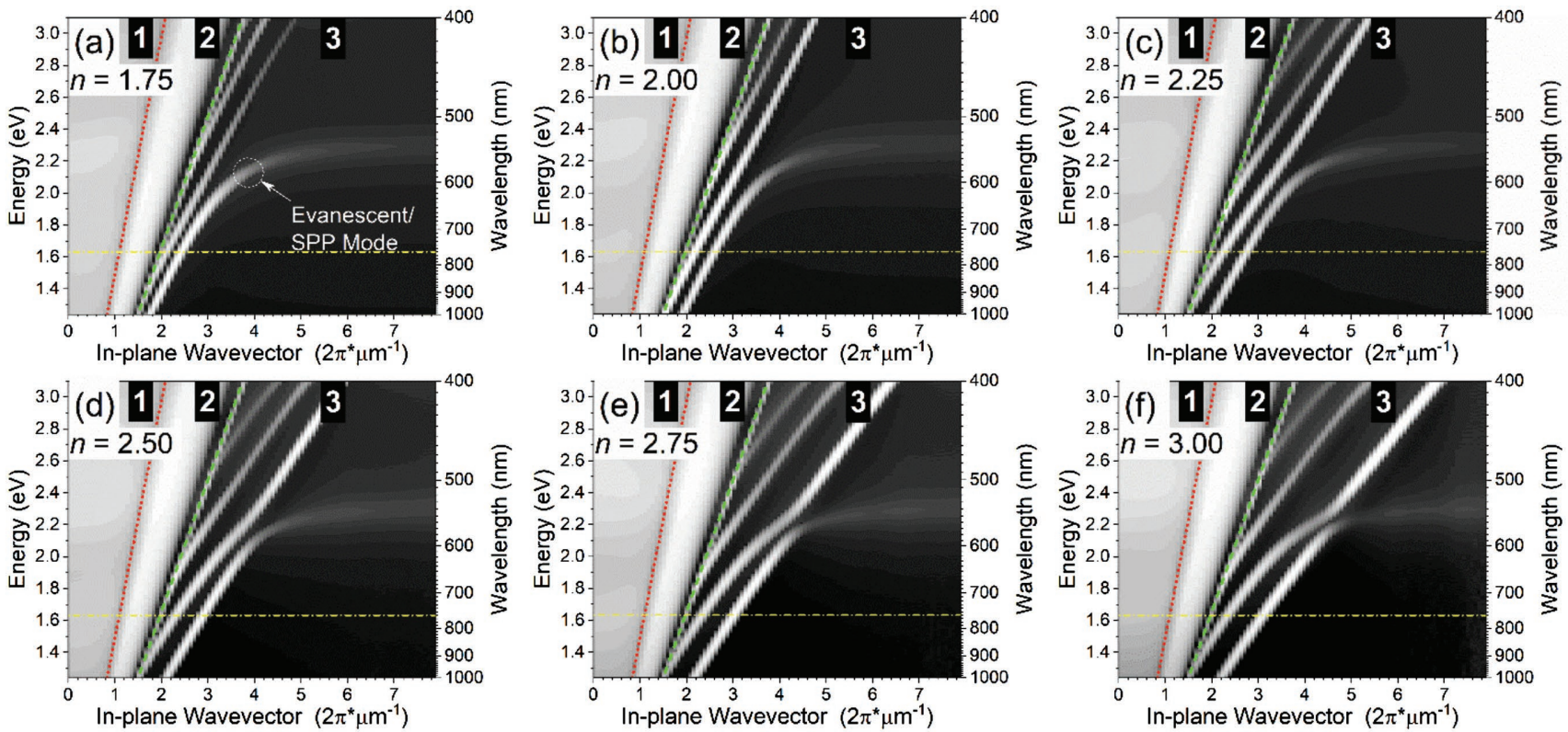

Figure 2. Dispersion diagrams of transverse magnetic (TM) polarized light as the function of the energy (or wavelength) and the in-plane wavevector of various refractive indices of the EML. The refractive indices of EML are a) $1.75, b) 2$, c) 2.25 , d) 2.50, e) 2.75, and f) 3.00 .

index of the EML increases, the waveguide modes $\left(\mathrm{TM}_{1}\right.$ and $\mathrm{TM}_{2}$ ) gradually shift toward the large in-plane wavevector. When the refractive index of the EML is larger than 2.5, the $\mathrm{TM}_{1}$ curve is significantly twisted at the energy between 2.1 and $2.5 \mathrm{eV}$ (corresponding to emissive wavelength between 500 and $600 \mathrm{~nm}$ ), and tends to overlap with the $\mathrm{TM}_{0}$ curve. This result suggests that the SPP mode and the waveguide mode begin to couple with each other to generate new hybrid TM modes. As the change of $\mathrm{TM}_{0}$ and $\mathrm{TM}_{1}$ modes around $760 \mathrm{~nm}$ (the central emissive wavelength of $\mathrm{MAPbI}_{3}$ ) are not as obvious as those between 500 and $600 \mathrm{~nm}$, the new hybrid TM modes do not definitely exist in PeLEDs based on perovskites such as MAPbI ${ }_{3}$. Further investigation on the full power dissipation spectra of these devices at $760 \mathrm{~nm}$ (Figure S2, Supporting Information) shows the same result. When the refractive index of the EML increases, the resonant peaks of $\mathrm{TM}_{0}$ and $\mathrm{TM}_{1}$ modes only shift slightly toward larger in-plane wavevectors.

To further study the coupling between the SPP mode and the waveguide mode in the PeLEDs with the infrared emission, we investigate the spatial distribution of power density of $\mathrm{TM}_{0}$ and $\mathrm{TM}_{1}$ modes (at $760 \mathrm{~nm}$ ) inside the devices where the EMLs have different refractive indices. As shown in Figure 3a, where the refractive index of the EML is 1.75 , the power intensity of $\mathrm{TM}_{0}$ decreases exponentially at the $\mathrm{MoO}_{3} / \mathrm{Au}$ interface. This is the critical feature of the SPP mode. ${ }^{[32]}$ The profile of $\mathrm{TM}_{1}$ is a typical sinusoid shape of the waveguide mode, ${ }^{[33]}$ which goes across the glass/ITO/ZnO layers in Figure 3a. Figure 3a,b shows bare coupling between the $\mathrm{TM}_{0}$ and $\mathrm{TM}_{1}$ modes. However, when the refractive index increases to 2.25 and above (Figure $3 \mathrm{c}-\mathrm{f}$ ), one sinusoid wave across the EML appears in the $\mathrm{TM}_{0}$ curves. This observation indicates that the SPP mode couples with the waveguide mode in the PeLEDs with refractive index of $>2.25$. Besides, the $\mathrm{TM}_{1}$ curves in Figure $3 \mathrm{c}-\mathrm{f}$ shows the feature of SPP mode at the $\mathrm{MoO}_{3} / \mathrm{Au}$ interface. Therefore, strong coupling between the waveguide mode and the SPP mode does exist in infrared PeLEDs (i.e., $\mathrm{MAPbI}_{3}$-based devices), resulting in two new hybrid TM modes and distinctive spatial power distribution. From the perspective of the light extraction, one nanostructure at the $\mathrm{ZnO} / \mathrm{EML}$ interface could be very efficient to extract light trapped by both the waveguide and the SPP modes at the same time for the PeLEDs with large$n$ EML because both modes have high power density near this interface.

To quantitatively analyze how the refractive index of the EML affects the $\eta$, the power distribution into different optical modes in the PeLEDs are calculated through the transfer matrix approach of a classical electroluminescent model. Figure 4 depicts the simulated power distribution in different optical modes of PeLEDs with various EMLs whose refractive indices change from 1.75 to 3.00 . As demonstrated above, the waveguide mode could couple with the SPP mode when the EML has high refractive index. Therefore, the power fraction of the waveguide and SPP modes are added together. In Figure 4a, where the refractive index of the EML is 1.75 , the $\eta$ hardly changes with the thickness of the EML, and shows its maximum at $\approx 16.7 \%$. This value is consistent with the theoretical calculation according to the ray-optics theory in LEDs based on organic emitters whose refractive index is $\approx 1.7 .{ }^{[20,34,35]}$ Interestingly, the $\eta_{\max }$, which is supposed to be smaller according to the ray-optics theory, does not reduce as the refractive index of the EML increases (Figure 4b-f). In contrast, it fluctuates around $17 \%$, and the $\eta_{\max }$ even increases to $25.1 \%$ when the refractive index reaches 2.50 or above (Figure $4 \mathrm{~d}-\mathrm{f}$ ). It is also interesting to note that larger refractive index makes the $\eta$ values more sensitive to the thickness of the EML, ranging between $11.5 \%$ and $25.1 \%$ when the refractive index is 2.50 (Figure $4 \mathrm{~d}$ ). Therefore, the large refractive index of perovskite materials will strengthen the interference of light inside the thin films. 


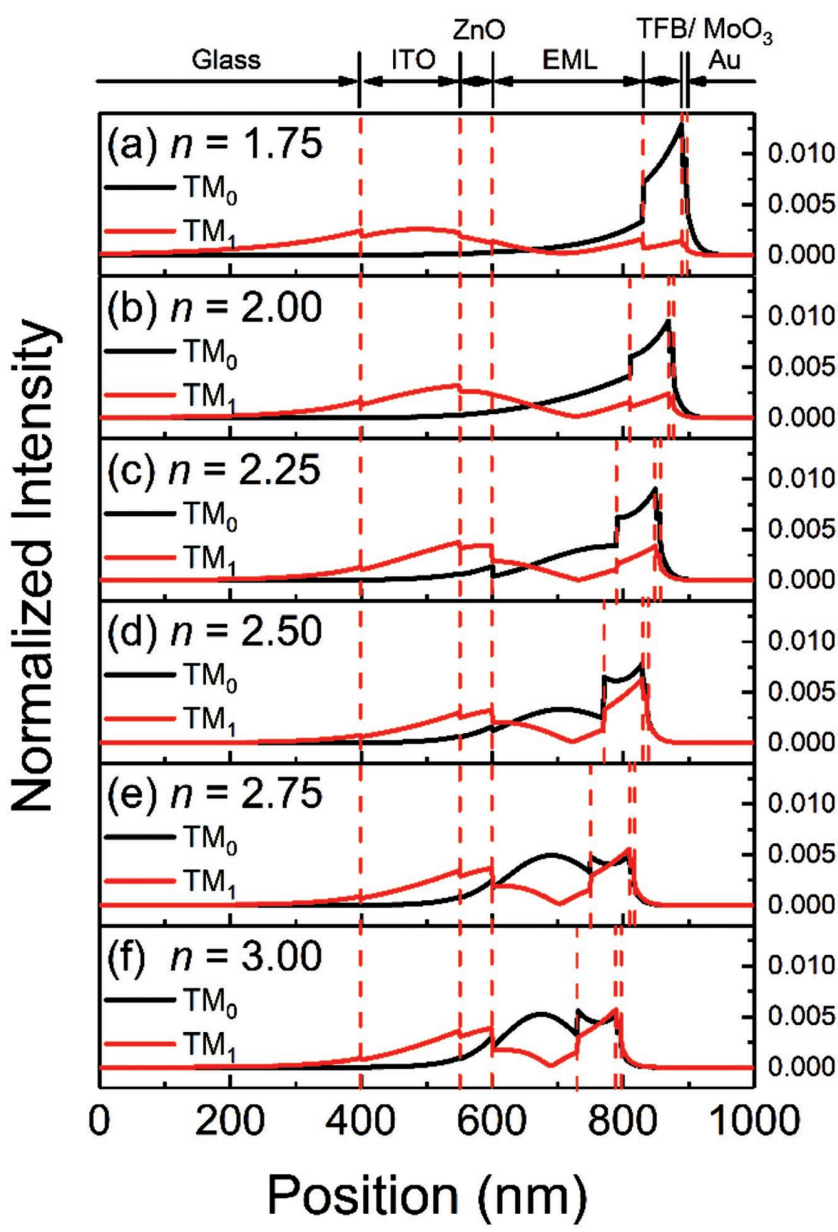

Figure 3. Spatial distribution in every functional layer of the power density of $\mathrm{TM}_{0}$ and $\mathrm{TM}_{1}$ modes at $760 \mathrm{~nm}$. The refractive indices are a) 1.75 , b) 2.00 , c) 2.25 , d) 2.50 , e) 2.75 , and f) 3.00 . The thicknesses of the EMLs used in the simulations are those resulting in the maximum light outcoupling efficiency, which are a) 230 , b) 210 , c) 190 , d) 170 , e) 150 , and f) $130 \mathrm{~nm}$, respectively.

It will directly affect the power fraction of waveguide and SPP modes. However, through careful design of the device structure (optimization of the thickness of each layer), a high $\eta$ of $\approx 25 \%$ can still be achieved.

In Figure 4 and related discussion, the refractive index of the EML is assumed constant over the emissive region. The power distribution into different optical modes in PeLEDs based on the real refractive indices of $\mathrm{MAPbI}_{3}$ and $\mathrm{NFPI}_{7}$ are presented in Figure 5 . The $\eta_{\max }$ values of $\approx 25.7 \%$ and $\approx 19.6 \%$ can be achieved in devices based on $\mathrm{MAPbI}_{3}$ and $\mathrm{NFPI}_{7}$, respectively. The latter is very close to the estimation value $(19.5 \%)$ from the experimental results. ${ }^{[14]}$ This simulating result indicates that PeLEDs based on 3D perovskites (i.e., $\mathrm{MAPbI}_{3}$ ) show relatively higher $\eta_{\max }$ values than those based on quasi-2D ones (i.e., $\mathrm{NFPI}_{7}$ ). However, the $\eta$ values of the 3D PeLEDs are more sensitive to the thickness of the EML (Figure 5a), suggesting that optimization of the thickness of the EML will not only influence the charge transport balance in the device but also change the $\eta$ values. Therefore, a compromise between the charge transport balance and the $\eta$ value should be taken into consideration to achieve high EQEs in 3D PeLEDs. In contrast, for quasi-2D PeLEDs, the $\eta$ value is hardly sensitive to the thickness of the EML (Figure $5 b$ ), indicating that the charge transport balance should be the primary concern when doing device engineering. From the observations of Figures 4 and 5, we can conclude that PeLEDs based on 3D perovskites with relatively larger refractive indices will have higher $\eta_{\max }$ values than those based on quasi-2D perovskites with smaller refractive indices. However, the $\eta$ values of 3D PeLEDs are highly dependent on the thickness of the EML, whereas they are almost invariant in quasi-2D PeLEDs.

The recombination zone of a LED could be located at either side of the EML. In addition to the above-mentioned case of TFB/EML interface, we also study the optical energy losses in PeLEDs when the recombination zone is located at the $\mathrm{ZnO} /$ EML interface. As shown in Figures S3 and S4 in the Supporting Information, the EQE variation with the thickness of the EML becomes larger for the cases based on smaller refractive index material and $\mathrm{NFPI}_{7}$. This is because the distance between the recombination zone and the metal electrode changes with the thickness of EML as well, which would arise serious optical destructive interference.

In conclusion, we have systematically studied the optical energy losses in PeLEDs by optical simulations and demonstrated how the large refractive index of perovskite materials influences the optical losses. The simulation results show that the refractive index of the EML does affect the light out-coupling efficiency $\eta$. However, high $\eta_{\max }$ values of $\approx 25 \%$ can be achieved in $\mathrm{MAPbI}_{3}$-based 3D PeLEDs through careful device engineering. For $\mathrm{NFPI}_{7}$-based quasi-2D PeLEDs, relatively lower $\eta_{\max }$ values of $\approx 20 \%$ can be achieved. Our results also suggest that a compromise between the charge transport balance and the $\eta$ value should be taken into consideration to achieve high-EQE 3D PeLEDs, because the larger refractive index will make the $\eta$ more sensitive to the thickness of the EML. However, for quasi-2D PeLEDs with relatively smaller refractive indices, the charge transport balance should be the primary concern when doing device engineering. In addition, we find that new hybrid TM modes appear and the different spatial power distribution could make it easier to enhance the $\eta$ through light extraction techniques. For example, it may be helpful to make the perovskite layer into nanostructures during its crystallization in the perspective of light extraction. Our results present primary understanding of the optical energy losses in PeLEDs and will spur new developments in the aspects of device engineering and light extraction techniques to achieve high-efficiency PeLEDs.

\section{Experimental Section}

Simulation Model: A classical electromagnetic model was adopted, treating the radiative decay of excitonic species as an ensemble of classical electrical dipole antennas radiating electromagnetic power. This was widely used in the simulation of the spontaneous emission inside a structure composed of stacked thin films. ${ }^{[22-27,35-38]}$

In the modeling, the following assumptions were made:

1) The dipole orientation of emitters is isotropic. Isotropic transition dipole moment is a common case in the discussion. The total radiated 

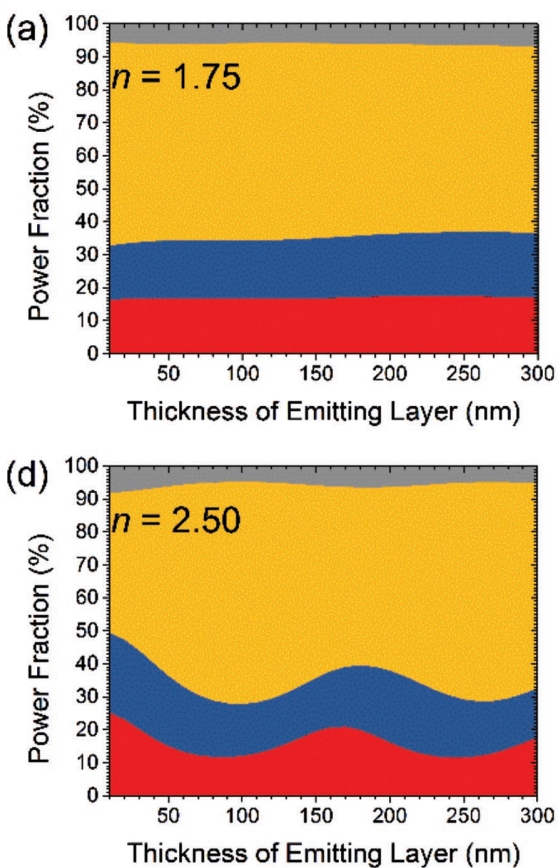

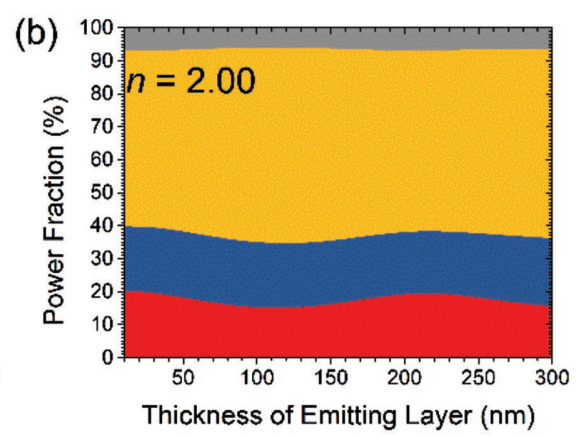

(e)

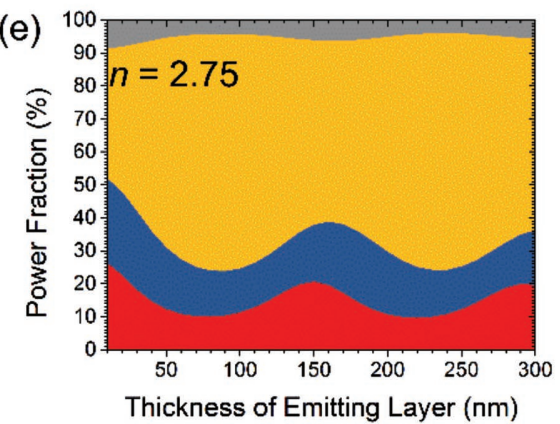

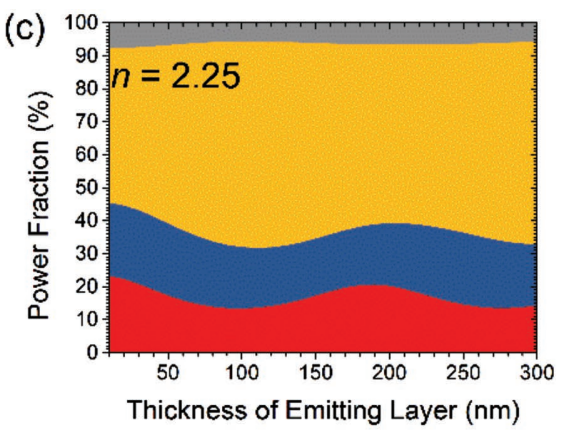

(f)

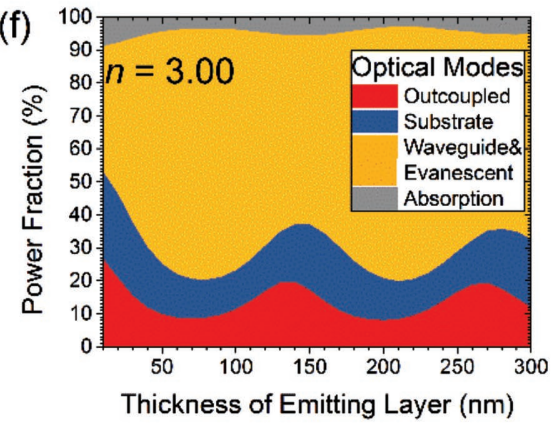

Figure 4. Power distribution in different optical modes of PeLEDs with various refractive indices of the EML. The refractive indices are a) 1.75 , b) 2.00 , c) 2.25 , d) 2.50 , e) 2.75 , and f) 3.00 . The recombination zone is located at the EML/TFB interface. The emissive spectrum is the same as that of MAPbI 3 .

power has a linear relationship with the fraction of the horizontal/ vertical dipoles as indicating by the following equation

$P_{\text {total }}=\alpha P_{\text {horizontal }}+(1-\alpha) P_{\text {vertical }}$

where $\alpha$ is the fraction of horizontal dipoles, which equals to $2 / 3$ in the isotropic case. In recent studies, ${ }^{[39-42]}$ the characterization of the angular dependent $p$-polarized PL emission spectra of the single emitting layer on a quartz substrate has been adopted to determine the orientation of the transition dipole moments. Higher outcoupling efficiency can be expected, if one perovskite material has more horizontal emitters.

2) The properties of all materials are isotropic. This is an intrinsic limitation of the electromagnetic model we use in this work. To deal with anisotropic materials, an effective refractive index is commonly used and it can be expressed as follows for uniaxial cases ${ }^{[33]}$ $\frac{1}{n_{\text {eff }}^{2}}=\frac{\cos ^{2} \theta}{n_{o}^{2}}+\frac{\sin ^{2} \theta}{n_{\mathrm{e}}^{2}}$

where $\theta$ is the angle between the propagation direction and the normal of the surface.

3) The emitting medium is nonabsorbing at the emissive region. This is also an intrinsic limitation of the electromagnetic model. Complexvalued refractive indices could result in numerical divergences in the calculation. For perovskite materials which have large Stocks-shift (i.e., weak overlap between the absorption spectrum and the luminescent spectrum), for example NFPI ${ }_{7}$, the assumption is valid ${ }^{[14]}$; For other perovskite materials, the description of the reabsorption effect can be included in the photoluminescent quantum efficiency. Because excitons from reabsorption will eventually decay through either radiative or nonradiative process. Therefore, it would not affect the calculation results of out-coupling efficiency seriously and can be treated
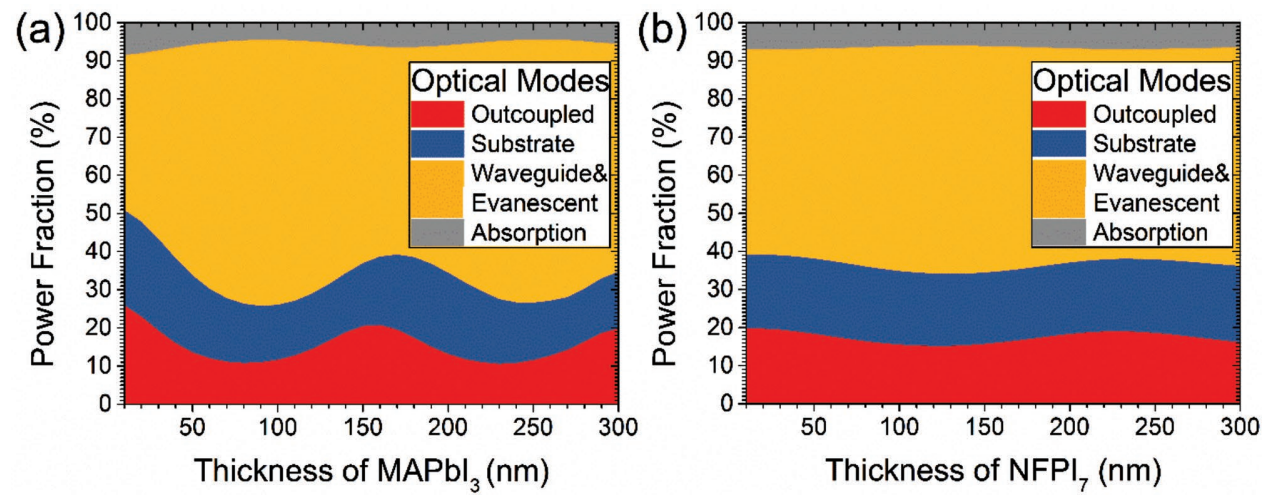

Figure 5. Power distribution in different optical modes of PeLEDs based on optical parameters of a) $\mathrm{MAPbl}_{3}$ and $\mathrm{b}$ ) $\mathrm{NFPI}_{7}$. The recombination zone is located at the EML/TFB interface. 
independently. A similar case is using the electromagnetic model to simulate the quantum-dot LEDs, ${ }^{[38]}$ while quantum dots usually have small Stokes-shift.

4) A discrete emitter position is used, i.e., an infinitely narrow distribution at the interface between the emitting layer and the charge-transporting layer. This is a common case due to the present lack of specific information for the distribution profile of emitters.

5) The devices are operated at low excitation levels or at low exciton concentrations in the emitting layer. This means exciton annihilation processes, e.g., Auger recombination, are not included in the discussions. Because relative physical models are unavailable or still under development. And this assumption can be satisfied in all real devices.

To obtain solutions of electromagnetic fields or power densities, two numerical calculation methods were adopted considering the time and data amount of the calculation tasks. Both of methods were well reported and widely used. First, developed for modelling of multilayer organic light-emitting diodes, a transfer matrix approach to describe radiating dipoles located in the emission layer was used, which was well described by Furno et. al. ${ }^{[28]}$ It was very convincing in the quantitative analysis of the radiated power from the dipoles. ${ }^{[28,36-38,44]}$ Another numerical calculation based on the finite-difference time-domain (FDTD) method ${ }^{[45-47]}$ was implemented. It was used in the analysis of the dispersion relation and spatial power distribution of various optical modes. ${ }^{[48-52]}$ In the FDTD model, the boundary conditions perpendicular and parallel to the interfaces were Bloch and perfect matching layers. The simulation time step was $0.00233507 \mathrm{fs}$ and the simulation time was $1000 \mathrm{fs}$. The mesh step was $0.5 \mathrm{~nm}$ over the device region and auto nonuniform mesh was used over other regions. An electric dipole was used as the light source.

Optical Modes in the Dispersion Diagrams: The in-plane wavevector in the medium is

$k_{\|}=k_{0} \cdot n \cdot \sin \theta$

where $k_{0}=2 \pi / \lambda$ is the wavevector in the vacuum. $\theta$ is the angle between the wavevector and the normal of the interface. As $0 \leq \theta \leq \frac{\pi}{2}$, each diagram in Figure 2 can be divided into three regions according to different refractive indices by the color lines. The red dot and green dash lines in Figure 2 are respectively corresponding to the maximum in-plane wavevector in the air $\left(k_{\|}=k_{0} \cdot n_{\text {air }}\right)$ and the glass substrate $\left(k_{\|}=k_{0} \cdot n_{\text {glass }}\right)$. Thus, Region 1 is the direct emission, which is the area between the vertical axis and the red dot line $\left(0 \leq k_{\|} \leq k_{0} \cdot n_{\text {air }}\right)$. This region represents the fraction of photons that can escape into the air from the forward hemisphere direction. Region 2 is the substrate mode, which is between the red dot line and the green dash line $\left(k_{0} \cdot n_{\text {air }} \leq k_{\|} \leq k_{0} \cdot n_{\text {glass }}\right)$. It shows the fraction of photons trapped inside the device due to the total internal reflection at the air/glass interface. The left $\left(k_{0} \cdot n_{\text {glass }} \leq k_{\|} \leq k_{0} \cdot n_{\text {eff }}\right)$ is Region 3 representing the waveguide mode and the evanescent/SPP mode. This region demonstrates photons trapped inside the thin films. The bright and dark regions denote the high and low power density, respectively.

Preparation of Perovskite Thin Films: $\mathrm{NFPI}_{7}$ precursor solutions were prepared by dissolving 1-naphthylmethylamine iodide (NMAI), formamidinium iodide (FAl), and $\mathrm{Pbl}_{2}$ with a molar ratio of 2:1:2 in dimethylformamide (DMF) (10 wt\%) and stirred at $60{ }^{\circ} \mathrm{C}$ for $2 \mathrm{~h}$ in a nitrogen-filled glovebox. The $\mathrm{NFPl}_{7}$ film was prepared by spin-coating the precursor solution onto a Si substrate at $4000 \mathrm{rpm}$ for $30 \mathrm{~s}$, followed by annealing on a hot plate at $100{ }^{\circ} \mathrm{C}$ for $10 \mathrm{~min}$ in the nitrogen-filled glovebox. The $\mathrm{MAPbl}_{3}$ precursor solution was synthesized by mixing $\mathrm{MAl}$ and $\mathrm{Pbl}_{2}$ in a 3:1 molar stoichiometric ratio in DMF to obtain a $0.5 \mathrm{~m}$ solution. The solution was spin-coated on a clean Si substrate at $4000 \mathrm{rpm}$ for $30 \mathrm{~s}$, to which $0.4 \mathrm{~mL}$ toluene was dropped after $10 \mathrm{~s}$ of spinning, followed by annealing on a hot plate at $100^{\circ} \mathrm{C}$ for $10 \mathrm{~min}$. The $\mathrm{MAPbBr}_{3}$ film was prepared in the same way as $\mathrm{MAPbl}_{3}$.

Characteristics: The refractive indices and thicknesses used in the simulations were measured by a variable angle spectroscopic ellipsometer (J. A. Woollam Co., Inc.). The spectra were collected at $35^{\circ}$, $45^{\circ}, 55^{\circ}$, and $65^{\circ}$. And all samples were spin-coated on the Si wafer and all spectra were fitted using the model of "Si with absorbing film."

\section{Supporting Information}

Supporting Information is available from the Wiley Online Library or from the author.

\section{Acknowledgements}

This work was supported by the ERC Starting Grant (717026), the Carl Tryggers Stiftelse, the European Commission Marie Skłodowska-Curie Actions (691210), the Swedish Government Strategic Research Area in Materials Science on Functional Materials at Linköping University (Faculty Grant SFO-Mat-LiU no. 2009-00971), and the National Basic Research Program of China-Joint Research Program between China and European Union (2016YFE0112000). X.-K.L. is a VINNMER and Marie Skłodowska-Curie Fellow (No. 2016-02051), and would like to acknowledge financial support from the State Key Laboratory of Luminescent Materials and Devices at South China University of Technology (No. 2017-skllmd-05) and the State Key Lab of Silicon Materials at Zhejiang University (No. SKL2017-03). Y.L. would like to thank financial support from the China Scholarship Council (No. 201506920047).

\section{Conflict of Interest}

The authors declare no conflict of interest.

\section{Keywords}

external quantum efficiency, light out-coupling efficiency, light-emitting diodes, optical energy loss, perovskites

Received: May 22, 2018

Revised: June 8, 2018

Published online: July 1, 2018

[1] M. A. Green, A. Ho-Baillie, H. J. Snaith, Nat. Photonics 2014, 8, 506.

[2] H. S. Jung, N. G. Park, Small 2015, 11, 10.

[3] T. C. Sum, N. Mathews, Energy Environ. Sci. 2014, 7, 2518.

[4] A. Sadhanala, S. Ahmad, B. Zhao, N. Giesbrecht, P. M. Pearce, F. Deschler, R. L. Z. Hoye, K. C. Gödel, T. Bein, P. Docampo, S. E. Dutton, M. F. L. De Volder, R. H. Friend, Nano Lett. 2015, 15, 6095.

[5] H. P. Kim, J. Kim, B. S. Kim, H.-M. Kim, J. Kim, A. R. b. M. Yusoff, J. Jang, M. K. Nazeeruddin, Adv. Opt. Mater. 2017, 5, 1600920.

[6] S. A. Veldhuis, P. P. Boix, N. Yantara, M. Li, T. C. Sum, N. Mathews, S. G. Mhaisalkar, Adv. Mater. 2016, 28, 6804.

[7] M. Sessolo, L. Gil-Escrig, G. Longo, H. J. Bolink, Top. Curr. Chem. 2016, 374, 52.

[8] S. Chen, C. Zhang, J. Lee, J. Han, A. Nurmikko, Adv. Mater. 2017, 29, 1604781

[9] T. Matsushima, S. Hwang, A. S. D. Sandanayaka, C. J. Qin, S. Terakawa, T. Fujihara, M. Yahiro, C. Adachi, Adv. Mater. 2016, 28 , 10275.

[10] C. Chen, X. Q. Zhang, G. Wu, H. Y. Li, H. Z. Chen, Adv. Opt. Mater. 2017, 5, 1600539.

[11] A. B. Yusoff, H. P. Kim, X. Li, J. Kim, J. Jang, M. K. Nazeeruddin, Adv. Mater. 2017, 29, 1602940.

[12] J.-P. Correa-Baena, M. Saliba, T. Buonassisi, M. Grätzel, A. Abate, W. Tress, A. Hagfeldt, Science 2017, 358, 739.

[13] Z.-K. Tan, R. S. Moghaddam, M. L. Lai, P. Docampo, R. Higler, F. Deschler, M. Price, A. Sadhanala, L. M. Pazos, D. Credgington, 
F. Hanusch, T. Bein, H. J. Snaith, R. H. Friend, Nat. Nanotechnol. 2014, 9, 687.

[14] N. Wang, L. Cheng, R. Ge, S. Zhang, Y. Miao, W. Zou, C. Yi, Y. Sun, Y. Cao, R. Yang, Y. Wei, Q. Guo, Y. Ke, M. Yu, Y. Jin, Y. Liu, Q. Ding, D. Di, L. Yang, G. Xing, H. Tian, C. Jin, F. Gao, R. H. Friend, J. Wang, W. Huang, Nat. Photonics 2016, 10, 699.

[15] Y. Yuan, Y. Hu, Y.-X. Zhang, J.-D. Lin, Y.-K. Wang, Z.-Q. Jiang, L.-S. Liao, S.-T. Lee, Adv. Funct. Mater. 2017, 27, 1700986.

[16] Y.-L. Shi, F. Liang, Y. Hu, X.-D. Wang, Z.-K. Wang, L.-S. Liao, J. Mater. Chem. C 2017, 5, 5372.

[17] F. Liang, Y. Liu, Y. Hu, Y. Shi, Y. Liu, Z.-K. Wang, X. Wang, B. Sun, L.-S. Liao, ACS Appl. Mater. Interfaces 2017, 9, 20239.

[18] C. Adachi, M. A. Baldo, M. E. Thompson, S. R. Forrest, J. Appl. Phys. 2001, 90, 5048.

[19] N. C. Greenham, R. H. Friend, D. D. C. Bradley, Adv. Mater. 1994, 6, 491.

[20] K. Saxena, V. K. Jain, D. S. Mehta, Opt. Mater. 2009, 32, 221.

[21] M. C. Gather, S. Reineke, J. Photonics Energy 2015, 5, 057607.

[22] W. Lukosz, J. Opt. Soc. Am. 1981, 71, 744.

[23] K. A. Neyts, J. Opt. Soc. Am. A 1998, 15, 962.

[24] W. L. Barnes, J. Mod. Opt. 1998, 45, 661.

[25] J. A. E. Wasey, W. L. Barnes, J. Mod. Opt. 2000, 47, 725.

[26] R. R. Chance, A. Prock, R. Silbey, J. Chem. Phys. 1974, 60, 2744.

[27] R. Chance, A. Prock, R. Silbey, Adv. Chem. Phys 1978, 37, 1.

[28] M. Furno, R. Meerheim, S. Hofmann, B. Lussem, K. Leo, Phys. Rev. B 2012, 85, 115205.

[29] J.-W. Lee, Y. J. Choi, J.-M. Yang, S. Ham, S. K. Jeon, J. Y. Lee, Y.-H. Song, E. K. Ji, D.-H. Yoon, S. Seo, H. Shin, G. S. Han, H. S. Jung, D. Kim, N.-G. Park, ACS Nano 2017, 11, 3311.

[30] Z. Xiao, R. A. Kerner, L. Zhao, N. L. Tran, K. M. Lee, T.-W. Koh, G. D. Scholes, B. P. Rand, Nat. Photonics 2017, 11, 108.

[31] L. Zhao, Y.-W. Yeh, N. L. Tran, F. Wu, Z. Xiao, R. A. Kerner, Y. L. Lin, G. D. Scholes, N. Yao, B. P. Rand, ACS Nano 2017, 11, 3957.

[32] J. M. Pitarke, V. M. Silkin, E. V. Chulkov, P. M. Echenique, Rep. Prog. Phys. 2007, 70, 1.

[33] P. K. Tien, Rev. Mod. Phys. 1977, 49, 361.

[34] K. Hong, J.-L. Lee, Electron. Mater. Lett. 2011, 7, 77.
[35] W. Brütting, J. Frischeisen, T. D. Schmidt, B. J. Scholz, C. Mayr, Phys. Status Solidi A 2013, 210, 44.

[36] Z. B. Wang, M. G. Helander, X. F. Xu, D. P. Puzzo, J. Qiu, M. T. Greiner, Z. H. Lu, J. Appl. Phys. 2011, 109, 053107.

[37] S. Nowy, B. C. Krummacher, J. Frischeisen, N. A. Reinke, W. Brütting, J. Appl. Phys. 2008, 104, 123109.

[38] R. Zhu, Z. Luo, S.-T. Wu, Opt. Express 2014, 22, A1783.

[39] C. Mayr, S. Y. Lee, T. D. Schmidt, T. Yasuda, C. Adachi, W. Brütting, Adv. Funct. Mater. 2014, 24, 5232.

[40] T.-A. Lin, T. Chatterjee, W.-L. Tsai, W.-K. Lee, M.-J. Wu, M. Jiao, K.-C. Pan, C.-L. Yi, C.-L. Chung, K.-T. Wong, C.-C. Wu, Adv. Mater. 2016, 28, 6976 .

[41] K.-H. Kim, J.-L. Liao, S. W. Lee, B. Sim, C.-K. Moon, G.-H. Lee, H. J. Kim, Y. Chi, J.-J. Kim, Adv. Mater. 2016, 28, 2526.

[42] T. Komino, Y. Oki, C. Adachi, Sci. Rep. 2017, 7, 8405.

[43] S.-Y. Kim, W.-I. Jeong, C. Mayr, Y.-S. Park, K.-H. Kim, J.-H. Lee, C.-K. Moon, W. Brütting, J.-J. Kim, Adv. Funct. Mater. 2013, 23, 3896.

[44] H. Shin, J.-H. Lee, C.-K. Moon, J.-S. Huh, B. Sim, J.-J. Kim, Adv. Mater. 2016, 28, 4920.

[45] A. Taflove, S. C. Hagness, Computational Electrodynamics: The Finite-difference Time-domain Method, Artech House, Norwood, Massachusetts 2005.

[46] A. Elsherbeni, V. Demir, The Finite Difference Time Domain for Electromagnetics: With Matlab Simulations, SciTech Publishing Incorporated, Raleigh 2016

[47] A. Jiménez-Solano, J. F. Galisteo-López, H. Míguez, J. Phys. Chem. Lett. 2018, 9, 2077.

[48] Y. Jin, J. Feng, X.-L. Zhang, Y.-G. Bi, Y. Bai, L. Chen, T. Lan, Y.-F. Liu, Q.-D. Chen, H.-B. Sun, Adv. Mater. 2012, 24, 1187.

[49] Y.-G. Bi, J. Feng, Y.-F. Li, X.-L. Zhang, Y.-F. Liu, Y. Jin, H.-B. Sun, Adv. Mater. 2013, 25, 6969.

[50] Q.-D. Ou, L. Zhou, Y.-Q. Li, S. Shen, J.-D. Chen, C. Li, Q.-K. Wang, S.-T. Lee, J.-X. Tang, Adv. Funct. Mater. 2014, 24, 7249.

[51] X.-B. Shi, M. Qian, D.-Y. Zhou, Z.-K. Wang, L.-S. Liao, J. Mater. Chem. C 2015, 3, 1666

[52] L. Zhou, Q.-D. Ou, Y.-Q. Li, H.-Y. Xiang, L.-H. Xu, J.-D. Chen, C. Li, S. Shen, S.-T. Lee, J.-X. Tang, Adv. Funct. Mater. 2015, 25, 2660. 